\title{
Modelado de la incertidumbre en proceso de producción utilizando emborronamiento no unitario y mapas borroso cognitivos: caso de estudio del aceite del oliva virgen.
}

\author{
P. Cano Marchal ${ }^{\dagger}$, C. Wagner ${ }^{\star}$, J. Gámez García ${ }^{\dagger}$, J. Gómez Ortega ${ }^{\dagger}$ \\ $\dagger$ Universidad de Jaén. Campus de Excelencia Internacional Agroalimentario (ceiA3). \\ ${ }^{\star}$ Lab for Uncertainty in Data and Decision Making (LUCID), School of Computer Science. University of Nottingham. \\ \{pcano, jggarcia, juango\}@ujaen.es, Christian.Wagner@nottingham.ac.uk
}

\section{Resumen}

Los sistemas de apoyo la decisión (DSS) son una herramienta útil para asistir a los operadores de planta en la selección de valores de referencias de proceso. Las entradas a este tipo de sistema para variables que no son fácilmente medibles en línea se realiza a menudo mediante la valoración de expertos, lo que conlleva un cierto grado de incertidumbre. La aplicación de lógica borrosa a estos DSS proporciona una manera sistemática de tratar esta incertidumbre en las variables. Este trabajo extiende el trabajo previo realizado utilizando Mapas Borrosos Cognitivos para la construcción de DSS e introduce una etapa de emborronamiento no unitario que proporciona una manera directa de considerar la incertidumbre en las entradas al sistema. El sistema propuesto está motivado por los desafíos que presenta la producción de aceite de oliva virgen de alta calidad.

Emborronamiento no unitario, Mapas Borrosos Cognitivos.

\section{Introducción}

El proceso de elaboración de aceite de oliva virgen (PEAOV) es un proceso industrial con bastantes variables relevantes y objetivos de producción contrapuestos. La relación de compromiso fundamental entre calidad del aceite de oliva virgen (AOV) obtenido y el agotamiento rendimiento de extracción - confiere una relevancia fundamental a la selección del objetivo de producción, dado que influye en gran manera en el rendimiento económico de la actividad.

La selección de las referencias de las variables de proceso es una etapa clave en el PEAOV, puesto que estos valores determinan la operación de la planta y, consecuentemente, la obtención de los objetivos de elaboración. Esta selección requiere considerar el proceso desde un punto de vista global, teniendo en cuenta las restricciones impuestas por las propiedades del fruto de entrada y la influencia de las variables de proceso.

En el PEAOV existen bastantes variables relevantes que son difíciles de medir, por lo que su valor suele ser estimado por operadores expertos. Las propiedades del fruto de entrada y la calidad organoléptica del AOV producido son algunos ejemplos de este tipo de variables. Esta dificultad tiene implicaciones desde dos puntos de vista: por un lado dificulta la construcción de modelos de proceso que se puedan utilizar para el diseño de controladores de alto nivel capaces de proporcionar el valor más adecuado de las referencias del proceso debido a que los datos experimentales son escasos y caros de obtener. Por otro lado, estas estimaciones conllevan de forma natural un cierto grado de incertidumbre acerca del valor verdadero de los parámetros estimados, inherente a la naturaleza aproximada y, hasta cierto punto, subjetiva de las valoraciones. Esta incertidumbre es a menudo aparente cuando más de un experto proporciona una valoración para la misma propiedad, puesto que rara vez coinciden exactamente.

En trabajos anteriores [3] se ha abordado el primer desafío proponiendo un sistema de apoyo a la decisión (DSS) capaz de sugerir referencias adecuadas para el PEAOV. La escasez de datos experimentales y el elevado número de variables de proceso y escenarios de producción condujo al diseño y construcción de un modelo del proceso basado en Mapas Borrosos Cognitivos (FCMs) [5], [10]. FCMs son una herramienta útil para modelar sistemas complejos con un elevado número de variables. Proporcionan una representación intuitiva de las relaciones entre las variables y permiten descomponer fácilmente el modelo en componentes más sencillos para abordar la construcción del mismo de una manera incremental.

En [3], se utilizó la solución de un problema de optimización que empleaba el modelo FCM como restricciones para proporcionar un conjunto de referencias de variables de proceso. La inclusión de un observador para las salidas permitía introducir realimentación en el sistema y sugerir ajustes de las referencias si el objetivo de elaboración no se estaba alcanzando realmente.

En este trabajo abordamos la segunda parte del problema y analizamos el modelado y el efecto de la incertidumbre sobre el proceso de toma de decisiones. La incertidumbre que afecta al valor de un parámetro en concreto se puede modelar utilizando conjuntos borrosos [8], que pueden, a su vez, ser utilizados como entradas al DSS, en lugar de introducir un valor nítido que no tendría en cuenta la información disponible sobre la incertidumbre. Los sistemas borrosos no unitarios han sido previamente 
explorados mostrando un elevando potencial en diversos contextos [6, 11, 1].

En particular, el objetivo de este trabajo es explorar las implicaciones de usar emborronamiento no unitario con el modelado basado en FCM y analizar su aplicación a un DSS simplificado para el PEAOV El resto del trabajo se organiza como sigue: la Sección 2 presenta algunos antecedentes del problema, tratando brevemente los FCM y el emborronamiento no unitario. Por su parte, la Sección 3 muestra el análisis de los FCM de emborronamiento no unitario en general, mientras que la Sección 4 trata su aplicación al DSS para el PEAOV. Finalmente, la Sección 5 incluye las conclusiones del trabajo.

\section{$2 \quad$ Antecedentes}

\subsection{Mapas Borrosos Cognitivos en el contexto de Sistemas Lógicos Borrosos}

Los Mapas Borrosos Cognitivos (FCM) son una metodología útil para modelar sistemas con un elevado número de variables implicadas, ya que facilita la obtención de conocimiento de los expertos permitiendo un enfoque sistemático.

El método propuesto se basa en los Simplified Dynamic Cognitive Networks (sDCN) [7]. Formalmente, el modelo se define como una tupla:

$$
\mathbf{M}=\langle\mathbf{V}, \mathbf{A}\rangle,
$$

donde $\mathbf{V}$ designa el conjunto de nodos - representando variables -,y A designa el conjunto de arcos que representan relaciones entre las variables.

Los nodos de la red pueden actuar como antecesores o sucesores, de acuerdo con su papel el proceso de cálculo. Los nodos antecesores son similares a la variables incluidas en los antecedentes de una regla difusa, mientras que los nodos sucesores se pueden identificar con las variables de la parte de consecuentes de la regla. La Figura (1) muestra un modelo de una capa genérico con varias entradas y una salida para un FCM singleton. Para modelos multicapas un nodo puede desempeñar ambos papeles, si existen arcos tanto que parten como que inciden en él.

Se definen las siguientes propiedades para cada nodo $v_{i} \in \mathbf{V}$ de la red:

- $U_{v_{i}}$ : el universo del discurso del nodo. Es el conjunto que contiene todos los posibles valores nítidos de la variable representada por $v_{i}$.

- $H_{v_{i}}$ : la colección de adjetivos $L_{v_{i}}^{k}$ definidos en $U_{v_{i}}$ :

$$
\begin{gathered}
L_{v_{i}}^{k}=\left\{\left\langle x, \mu_{L_{v_{i}}^{k}}(x)\right\rangle: x \in U_{v_{i}}\right\}, \\
H_{v_{i}}=\left\{L_{v_{i}}^{k}, k=1,2, \cdots, K_{i}\right\} .
\end{gathered}
$$

Aquí, $K_{i}$ representa el número total de conjuntos definidos en el nodo.

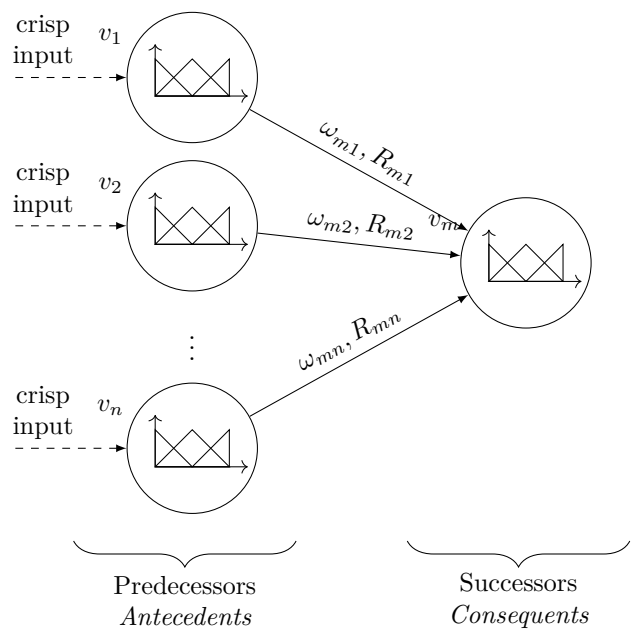

Figura 1: Modelo génerico con múltiples entradas y una única salida para FCM de emborronado unitario. Un modelo más complejo se puede construir componiendo esta estructura elemental.

- $S_{f}\left(v_{i}\right)$ : un vector que contiene el grado de pertenencia a cada conjunto borroso $L_{v_{i}}$ de un $v_{i}$ dado para una entrada nítida (ver Fig. 1).

$$
S_{f}\left(v_{i}\right)=\left[\mu_{L_{v_{i}}^{1}}, \cdots, \mu_{L_{v_{i}}^{K_{i}}}\right]^{T} .
$$

- $S_{c}\left(v_{i}\right)$ : el valor nítido asignado al nodo. Si $v_{i}$ actúa como sucesor, este valor se calcula utilizando el método definido por las Ecuaciones (6)-(8). Si $v_{i}$ actúa como antecedente, este valor que considera que ya está disponible, bien proporcionado por una entrada externa al nodo - si el nodo no tiene ningún antecesor - bien previamente calculado.

Por su parte, para cada arco $a_{i j}$ se definen las siguientes propiedades:

- $\omega_{i j}$ : intensidad de la relación entre $\operatorname{los} \operatorname{nodos} v_{i}$ y $v_{j}$.

- $R_{i j}$ : matriz de relación causal. Las entradas de esta matriz deben ser no negativas y definen la relación entre los conjuntos borroso de los nodos antecesor y sucesor conectados por el arco. El tamaño de la matriz es $K_{i} \times K_{j}$, donde $K_{i}$ y $K_{j}$ son el número de adjetivos en $H_{v_{i}}$ y $H_{v_{j}}$ respectivamente.

Estas propiedades de los arcos $\omega_{i j}$ y $R_{i j}$ desempeñan un papel similar a las reglas en un FLS basado en reglas, dado que estos son los elementos que codifican las relaciones entre las distintas variables del sistema. En la matriz $R_{i j}$ cada fila está asociada con un conjunto borroso definido en $U_{v_{i}}$ y cada columna lo está con uno definido en $U_{v_{j}}$. Cada entrada de $R_{i j}$ se puede considerar como una regla relacionando el valor del antecesor (nodo $v_{j}$ ) con el sucesor 
$\left(v_{i}\right)$, donde el peso de la regla viene dado por el valor de la entrada multiplicada por el peso asociado al arco.

Como ejemplo, consideremos la siguiente matriz:

$$
R_{i j}=\left[\begin{array}{lll}
0 & 0 & 1 \\
0 & 1 & 0 \\
1 & 0 & 0
\end{array}\right]
$$

La forma de la matriz denota que se han definido tres conjuntos borrosos tanto en $U_{v_{i}}$ como en $U_{v_{j}}$, y que las reglas asociadas son:

$$
\begin{aligned}
& \text { Si } v_{j} \text { es } L_{v_{j}}^{1} \text { Entonces } v_{i} \text { es } q_{i}^{3} \text {, con peso } \omega_{i j} \text {. } \\
& \text { Si } v_{j} \text { es } L_{v_{j}}^{2} \text { Entonces } v_{i} \text { es } q_{i}^{2} \text {, con peso } \omega_{i j} \text {. } \\
& \text { Si } v_{j} \text { es } L_{v_{j}}^{3} \text { Entonces } v_{i} \text { es } q_{i}^{1} \text {, con peso } \omega_{i j} .
\end{aligned}
$$

El cálculo del valor de un sucesor, dado el valor de sus antecesores, se calcula como sigue:

1. El impacto recibido por el nodo $i$ se define como:

$$
\mathbf{w}_{i}=\sum_{j=1}^{n_{i}} \omega_{i j} R_{i j} S_{f}\left(v_{j}\right)=\left[w_{i}^{1} w_{i}^{2} \cdots w_{i}^{K_{i}}\right]^{T}
$$

2. El cálculo del valor nítido $S_{c}\left(v_{i}\right)$ del nodo se realiza utilizando una combinación ponderada de los valores de pico de cada conjunto borroso, utilizando el impacto recibido por el nodo:

$$
S_{c}\left(v_{i}\right)=\frac{\sum_{k=1}^{K_{i}} w_{i}^{k} q_{i}^{k}}{\sum_{k=1}^{K_{i}} w_{i}^{k}} .
$$

3. Finalmente, el vector $S_{f}\left(v_{i}\right)$ captura la pertenencia cada adjetivo:

$$
S_{f}\left(v_{i}\right)=\left[\mu_{L_{v_{i}}^{1}}\left(S_{c}\left(v_{i}\right)\right) \mu_{L_{v_{i}}^{2}}\left(S_{c}\left(v_{i}\right)\right) \cdots \mu_{L_{v_{i}}^{K_{i}}}\left(S_{c}\left(v_{i}\right)\right)\right]^{T} .
$$

La Ecuación (7) realiza los procesos de inferencia y desemborronado de forma simultánea, y es similar a un sistema de Takagi-Sugeno-Kang (TSK) de orden cero.

\subsection{Emborronado no unitario}

El empleo de conjuntos borrosos como entradas a los FLS requiere definir un método para determinar el grado de disparo de las reglas basado en la interacción de los conjuntos que representan la entrada y el antecedente de cada regla. Un enfoque común es utilizar la interacción sup-min. Si denotamos el conjunto borroso de la entrada y del antecedente como $X$ y $L$ respectivamente, entonces:

$$
\mu_{X \circ L}=\sup _{u \in U_{X}} \min \left[\mu_{X}(u), \mu_{L}(u)\right] .
$$

La Figura (2) representa la intersección de de conjuntos borrosos Gaussianos con valores crecientes de desviación típica $(\sigma)$ - representado valores crecientes de incertidumbre

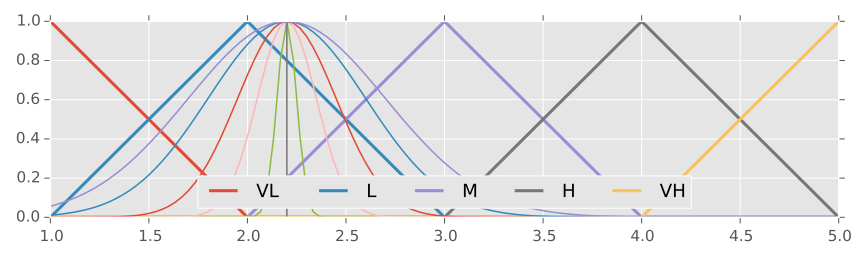

Figura 2: Intersección de Gaussianas de valores crecientes de $\sigma$ con funciones de pertenencia triangulares. Gaussianas con valores mayores de $\sigma$ intersectan más adjetivos, y a mayores valores de pertenencia.

sobre la entrada - con una colección de conjuntos borrosos triangulares. Como se ve en la Figura, los conjuntos borrosos con un mayor grado de incertidumbre intersectan más conjuntos triangulares - lo que se corresponde con un mayor número de reglas activadas - y a un mayor nivel de activación.

\section{Mapas Borrosos Cognitivos No Unitarios}

La utilización de emborronamiento no unitario en FCM implica considerar que las entradas a los nodos en la primera capa $\left(v_{j}\right)$ del modelo no son valores nítidos $\left(x_{j}\right)$, sino conjuntos borrosos $\left(X_{j}\right)$. Consecuentemente, el cálculo de $S_{f}\left(v_{j}\right)$ se debe llevar a cabo teniendo en cuenta la interacción de $X_{j}$ y el conjunto borroso asociado con cada una de los elementos de $L_{v_{j}}^{k}$. Si elegimos la interacción sup-min, entonces los elementos de $S_{f, n s}\left(v_{j}\right)$ se pueden calcular como:

$$
S_{f, n s}^{k}\left(v_{j}\right)=\sup _{u \in U_{v_{j}}} \min \left[\mu_{X_{j}}(u), \mu_{L_{v_{j}}^{k}}(u)\right]
$$

La columna izquierda de la Fig. 3 muestra un gráfico del valor de los elementos de $S_{f}\left(v_{j}\right)$ cuando $X_{k}$ es un conjunto borroso Gaussiano cuya media corresponde al valor del eje $x$ para tres valores de $\sigma$. Como se ve en la Figura, para los casos en que $\sigma>0$, los elementos de $S_{f}\left(v_{j}\right)$ no suman uno (i.e., para algunos cortes verticales, la suma de las pertenencias a las distintas MFs en mayor que uno); de hecho, cuanto mayor es $\sigma$, mayor es el valor de esta suma. Como se ha comentado en la Sección anterior, esta es una característica conocida del emborronamiento no unitario.

Para el caso de FCM tradicional, los conjuntos borrosos definidos en el universo de discurso de los nodos se eligen de forma que constituyan una partición borrosa, de forma que esté garantizado que la suma de todos los elementos de $S_{f}$ sea uno. Con el emborronamiento no unitario esta propiedad no está garantizada. Si embargo, para restablecerla, se puede renormalizar los valores como sigue:

$$
S_{f, n s}\left(v_{j}\right)=c_{j} \hat{S}_{f, n s}\left(v_{j}\right)
$$

con $c_{j}=\sum_{k} S_{f, n s}^{k}\left(v_{j}\right)$ y $\sum_{i} \hat{S}_{f, n s}^{k}=1$. De este modo, el impacto ejercido por el nodo $v_{j}$ sobre el nodo $v_{i}$ se puede 

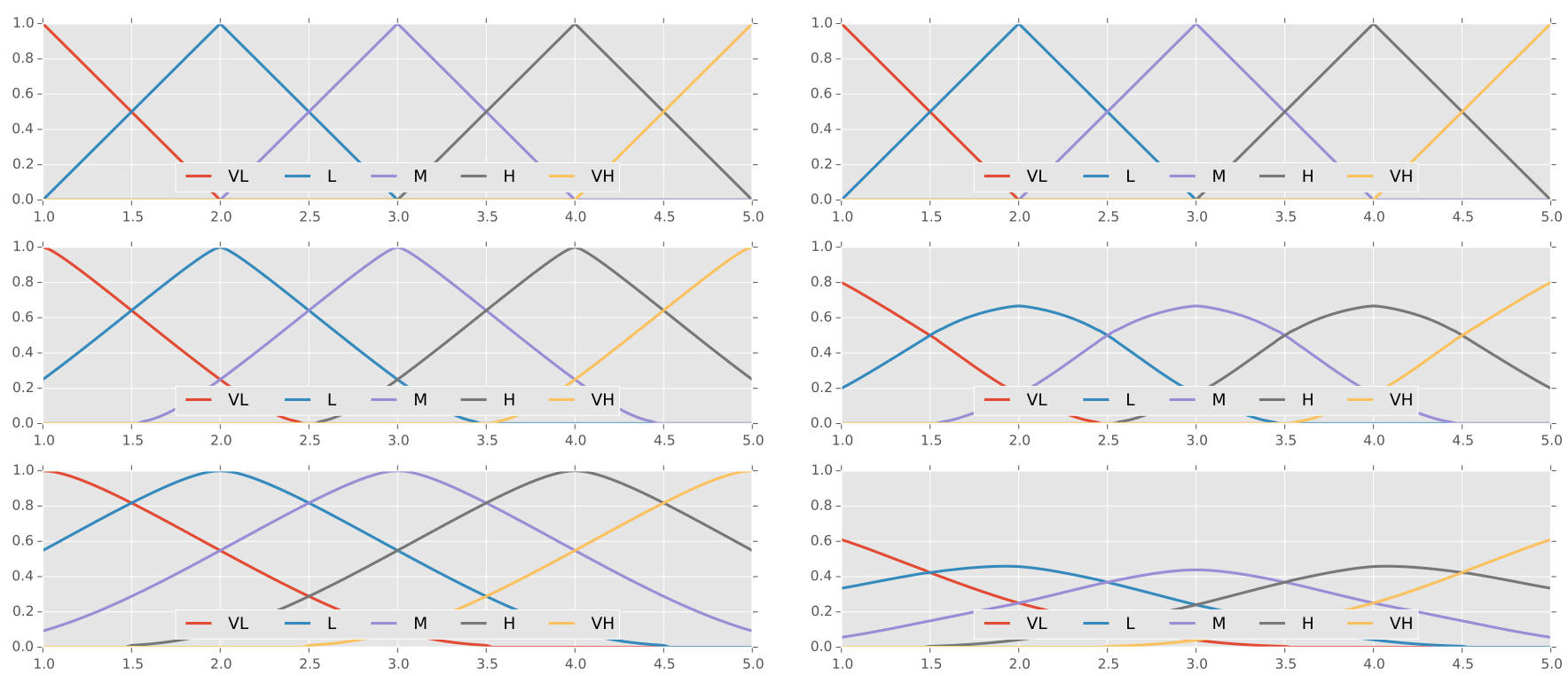

Figura 3: Comparación de $S_{f, n s}\left(u_{k}\right)$ (izquierda) y $\hat{S}_{f, n s}\left(u_{k}\right)$ (derecha) para $S_{f, n s}^{k}\left(v_{j}\right)=\sup _{u \in U_{v_{j}}} \min \left[\mu_{X_{j}}(u), \mu_{L_{v_{j}}^{k}}(u)\right]$ y $\sigma=[0,0.25,0.5]$ (arriba hacia abajo).

expresar como:

$$
\omega_{i j} R_{i j} S_{f, n s}\left(v_{j}\right)=\omega_{i j} R_{i j} c_{j} \hat{S}_{f, n s}\left(v_{j}\right)=\hat{\omega}_{i j} R_{i j} \hat{S}_{f, n s}\left(v_{j}\right)
$$

Esta Ecuación se puede interpretar de acuerdo con el significado de los diferentes elementos del modelo FCM. En esta metodología, la influencia de un nodo $v_{j}$ en su sucesor $v_{i}$ se puede separar en dos aspectos:

- Primero, la matriz de relación que conecta ambos nodos $\left(R_{i j}\right)$ define qué reglas se deben activar de acuerdo con los valores de $S_{f}\left(v_{i}\right)$. Esto se puede interpretar como una definición de qué valor debería tener el nodo atendiendo en exclusiva al valor de ese nodo de entrada en particular.

- Segundo, el peso $\omega_{i j}$ representa la intensidad de la influencia de $v_{j}$ relativa a todos los otros nodos que afectan al sucesor. Esto es, define la influencia en valor final de $v_{i}$ del valor propuesto por $v_{j}$.

La sustitución de $S_{f}\left(v_{j}\right)$ por $\hat{S}_{f, n s}\left(v_{j}\right)$ se puede interpretar intuitivamente como cambios en la consideración de la influencia del nodo $v_{j}$ debido a la incertidumbre en su valor. La columna derecha de Fig. 3 muestra gráficos de los valores de los elementos de $\hat{S}_{f, n s}\left(v_{j}\right)$ para los valores correspondientes de $S_{f, n s}\left(v_{j}\right)$ representados a la izquierda. Como se puede ver en el gráfico, el incremento de la incertidumbre provoca un descenso en cada componente de $\hat{S}_{f, n s}\left(v_{j}\right)$ y que un mayor número de elementos tengan valor distinto de cero. Esto significa que más reglas se activan, pero a menor nivel de certidumbre cada una de ellas.
Por su parte, la conversión de $\omega_{i j}$ en $\hat{\omega}_{i j}$ puede verse como un cambio en los pesos relativos del sistema. Como se ha comentado anteriormente, alta incertidumbre se corresponde con alto $c_{j}$, y, consecuentemente, mayores valores de $\hat{\omega}_{i j}$. Esto significa que valores cuyo valor es más incierto son asignados mayor influencia en el modelo, lo que no es deseable. Esto se puede corregir simplemente ignorando $c_{j}$ en el modelo. De este modo, el cálculo del impacto del nodo $v_{i}$ sobre $v_{j}$ se define como:

$$
\mathbf{w}_{i j}=\omega_{i j} R_{i j} \hat{S}_{f, n s}\left(v_{j}\right) .
$$

Esta ecuación se beneficia del efecto de suavizado correspondiente a la mayor dispersión de $\hat{S}_{f, n s}\left(v_{j}\right)$ (inherente al emborronamiento no unitario) sin alterar artificialmente los pesos del modelo. La siguiente Sección presenta y discute los resultados obtenidos al considerar Eq. (12) y (13) en los cálculos.

\section{Aplicación de FCM No Unitarios al PEAOV}

Trabajos previos han tratado sobre la construcción de un DSS para el PEAOV capaz de sugerir los valores de las referencias de proceso que maximizan una cierta función objetivo, sujetos a la características del fruto de entrada [3]. El DSS consiste de un modelo FCM del PEAOV que actúa como un conjunto de restricciones para el problema de optimización. La Figura 4 muestra un diagrama que representa un modelo simplificado del PEAOV utilizado para el DSS.

A pesar de que el principal objetivo del DSS es proporcionar las referencias de proceso, que vienen dadas por la 


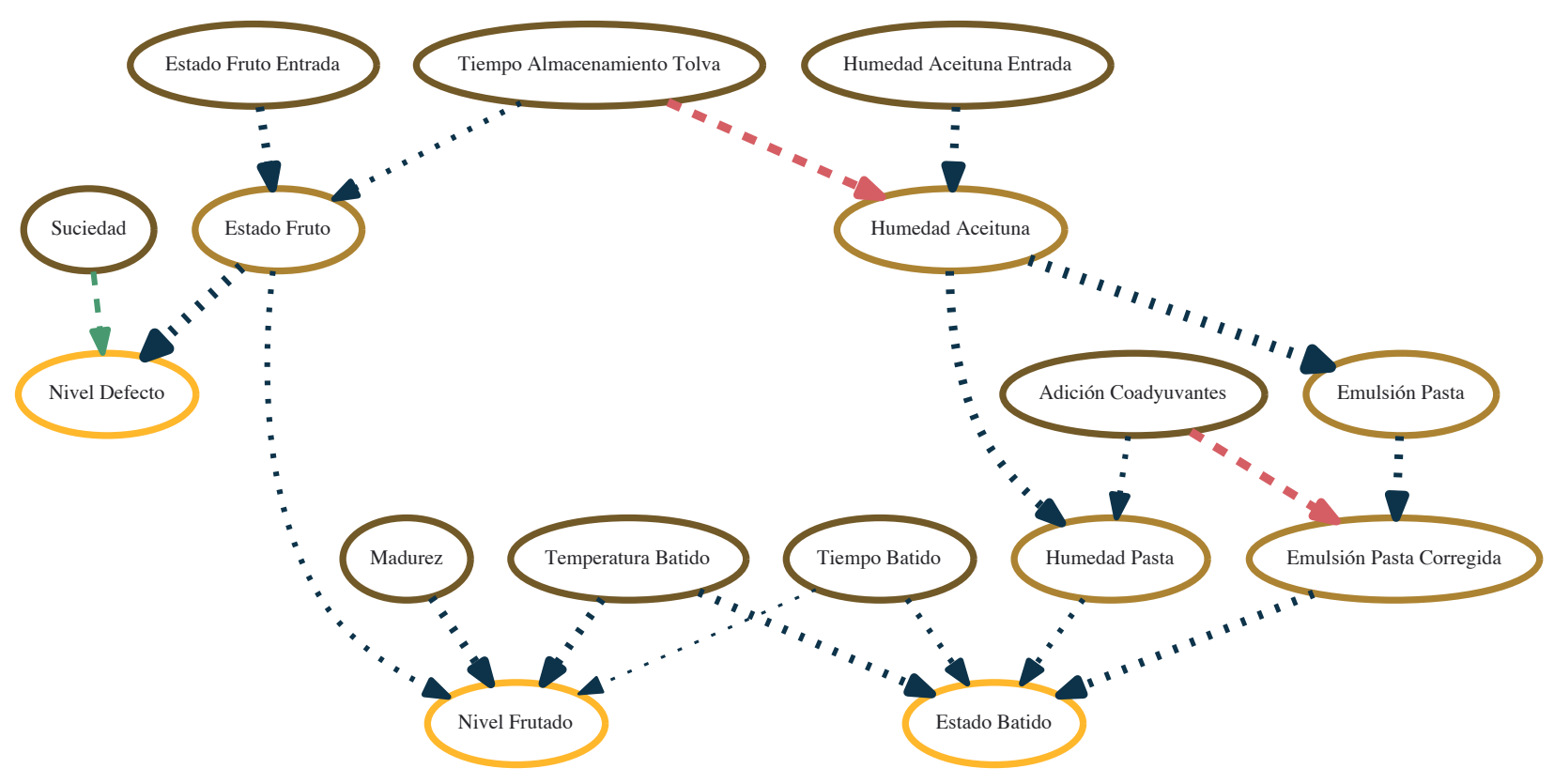

Figura 4: Modelo del PEAOV.

solución del problema de optimización, es de interés analizar la influencia del enfoque de emborronamiento no unitario sobre las variables de salida del modelo.

La Figura 5 muestra Estado de Batido en función de $\mathrm{Hu}$ medad Fruto de Entrada y Adición de Coadyuvantes para tres niveles de incertidumbre en Humedad Fruto de Entra$d a$ - izquierda a derecha - y empleando tanto el enfoque normalizado $\hat{S}_{f_{, n f}}$ y el no normalizado - arriba y abajo, respectivamente. Por su parte, Tiempo de Almacenamiento en Tolva, Tiempo de Batido y Temperatura de Batido son fijos. La inclusión de la incertidumbre modifica ligeramente la forma del gráfico, particularmente para valores altos de Humedad Fruto de Entrada. No hay, sin embargo, diferencia visible entre los enfoques normalizado y no normalizado.

La Figura 6 muestra la dependencia de Estado de Batido con Humedad Fruto de Entrada y Tiempo de Almacenamiento en Tolva. En este gráfico las diferentes implicaciones de la utilización del enfoque normalizado frente al no normalizado se hacen visibles, ya que valores crecientes de incertidumbre provocan efectos opuestos en la zona de valores alto de Humedad Fruto de Entrada. Se puede apreciar también la similitud de ambos enfoques para valores muy bajos de Tiempo de Almacenamiento en Tolva.

Centramos ahora nuestra atención en el DSS completo. Las variables del modelo se pueden clasificar según su papel en el problema de optimización como:
- Parámetros (p): variables cuyo valor es fijo, i.e., constantes. Estas incluyen tanto las propiedades del fruto de entrada como otros parámetros que puedan considerarse constantes.

- Salidas (y): variables que conforman la salida del proceso. En el modelo de la Figura 4 , estas variables son Frutado, Estado de Batido y Defecto.

- Nodos de decisión (u): estas son las variables de proceso cuyo valor constituye la salida del DSS, i.e., las referencias sugeridas al operador.

Consideremos el problema de hallar el valor óptimo de las variables de decisión u que permiten obtener un cierto valor de las salidas y para un cierto escenario de producción caracterizado por los valores de p. Este problema se puede formalizar como:

$$
\begin{array}{ll}
\underset{\mathbf{u}_{k}}{\operatorname{minimizar}} & J=(\mathbf{y}-\mathbf{T})^{T} Q(\mathbf{y}-\mathbf{T})+\mathbf{u}^{T} R \mathbf{u} \\
\text { sujeto a } & \mathbf{y}=f(\mathbf{u}, \mathbf{p}) \\
& \mathbf{p}=\mathbf{p}^{0} \\
& \mathbf{u}_{\min } \leq \mathbf{u} \leq \mathbf{u}_{\max }
\end{array}
$$

Aquí $\mathbf{p}^{0}$ representa los valores de $\mathbf{p}$ para la situación en concreto, $\mathbf{T}$ representa los valores de $\mathbf{y}$ prescritos y $f(\cdot)$ denota el modelo FCM. El peso relativo de las distintas 


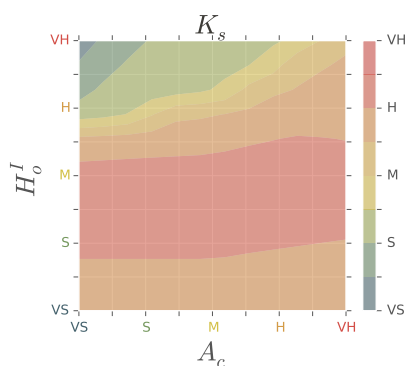

(a) Normalizado, $\sigma=0.05$

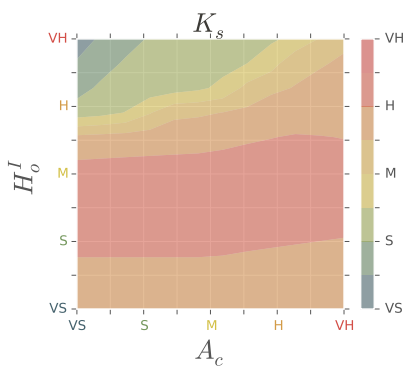

(d) No Normalizado, $\sigma=0.05$

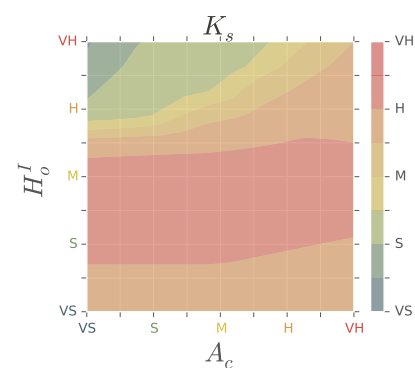

(b) Normalizado, $\sigma=0.25$

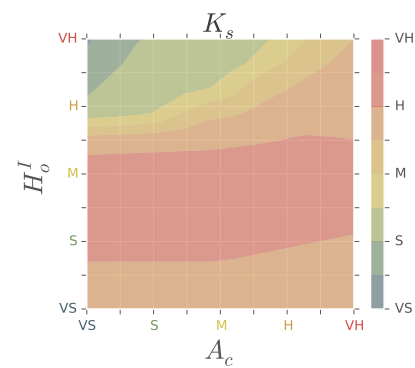

(e) No Normalizado, $\sigma=0.25$

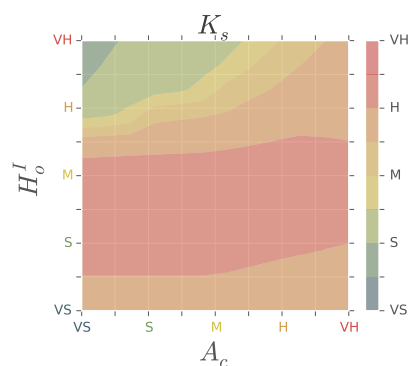

(c) Normalizado, $\sigma=0.5$

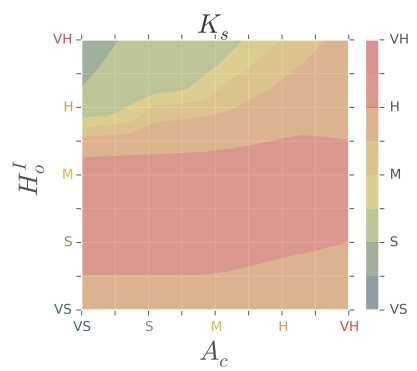

(f) No Normalizado, $\sigma=0.5$

Figura 5: Estado de Batido en función de Adición de Coadyuvantes (eje x) y Humedad Fruto de Entrada (eje y) para $\sigma=[0.05,0.25,0.5]$ (left to right). Las filas superior e inferior representan el enfoque normalizado y no normalizado, respectivamente.

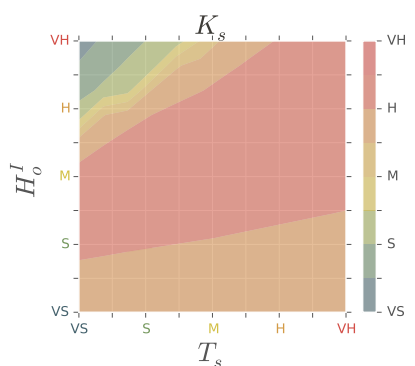

(a) Normalizado, $\sigma=0.05$

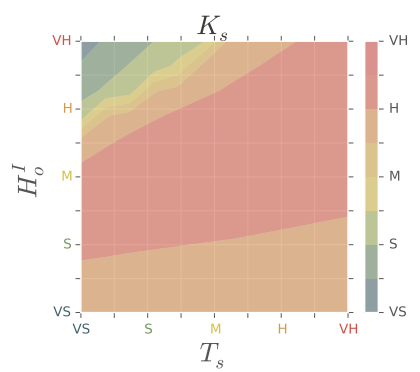

(d) No Normalizado, $\sigma=0.05$

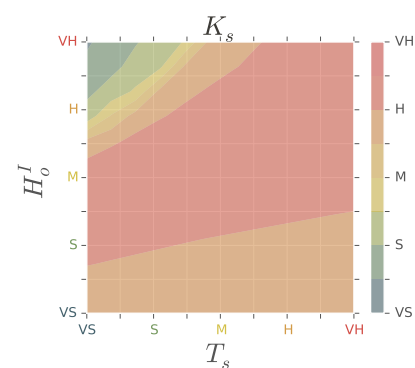

(b) Normalizado, $\sigma=0.25$

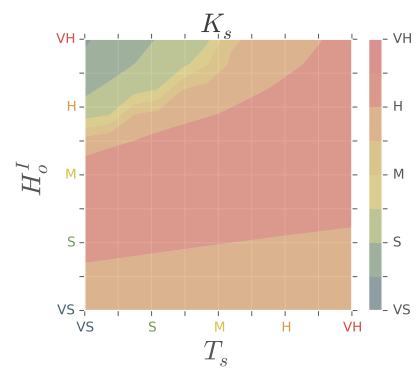

(e) No Normalizado, $\sigma=0.25$

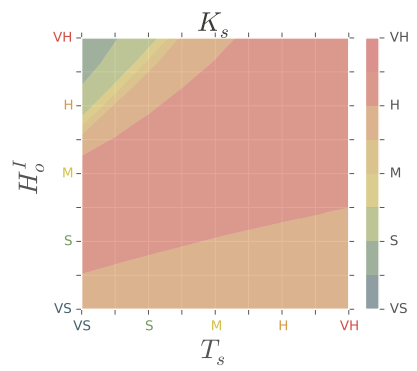

(c) Normalizado, $\sigma=0.5$

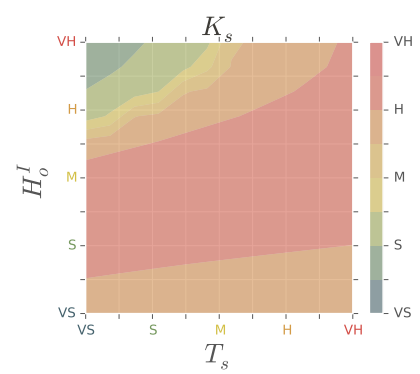

(f) No Normalizado, $\sigma=0.5$

Figura 6: Estado de Batido en función de Tiempo de Almacenamiento en Tolva (eje $x$ ) de Humedad Fruto de Entrada (eje $y$ ) for $\sigma=[0.05,0.25,0.5]$ (left to right). Las filas superior e inferior representan el enfoque normalizado y no normalizado, respectivamente. 


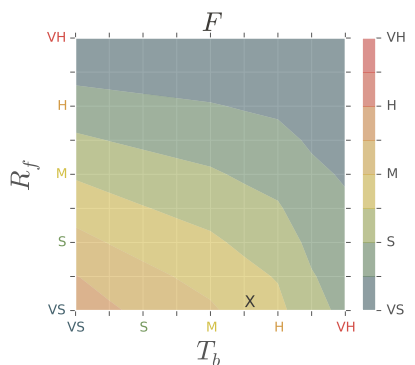

(a) Normalizado, $\sigma=0.05$

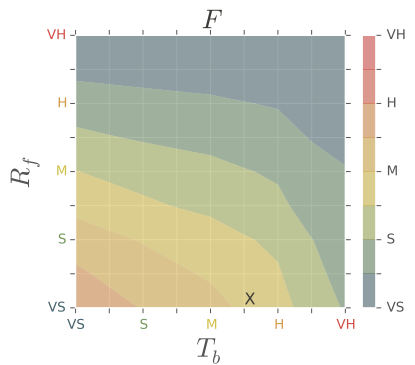

(d) No Normalizado, $\sigma=0.5$

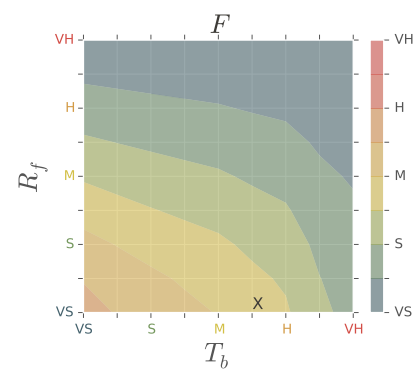

(b) Normalizado, $\sigma=0.25$

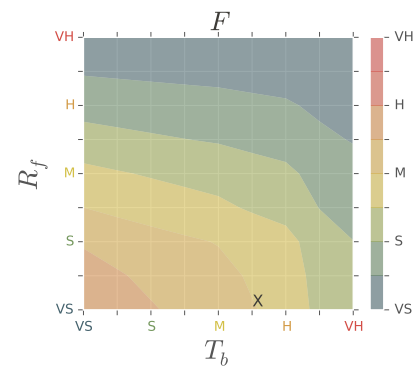

(e) No Normalizado, $\sigma=0.25$

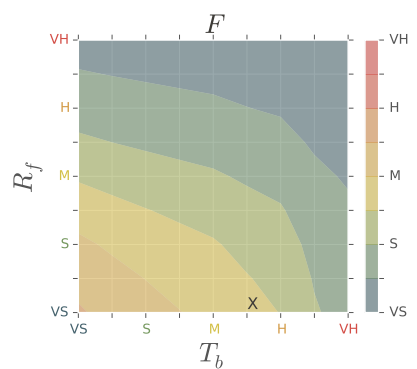

(c) Normalizado, $\sigma=0.5$

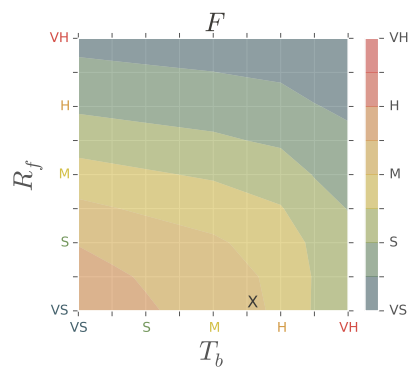

(f) No Normalizado, $\sigma=0.5$

Figura 7: Frutado en función de Tiempo de Batido (eje $x$ ) y Madurez (eje y) para $\sigma=[0.05,0.25,0.5]$ (lenta to right). Las filas superior e inferior representan el enfoque normalizado y no normalizado, respectivamente. La X marca una región en que se pone de manifiesto en incremento de peso asignado a Madurez en el enfoque no normalizado.

Cuadro 1: Referencias y valores de salida esperados para el modelado no unitario con normalización de $S_{f}^{n f}$ para valores dados de $\sigma$.

\begin{tabular}{lllll}
$\sigma$ & 0 & 0.05 & 0.25 & 0.5 \\
\hline Madurez & 4.00 & 4.00 & 4.00 & 4.00 \\
Frutado & 3.95 & 3.95 & 3.91 & 3.93 \\
Estado de Batido & 3.06 & 2.98 & 2.85 & 2.61 \\
Defecto & 1.00 & 1.00 & 1.00 & 1.00 \\
Tiempo de Batido & 3.87 & 3.26 & 3.00 & 3.00 \\
Temperatura de Batido & 2.00 & 2.00 & 1.90 & 1.62
\end{tabular}

salidas y el costo de las variables de decisión se define utilizando las matrices $Q$ y $R$ respectivamente, como es usual en Model Predictive Control [2].

Consideremos un escenario de producción en el que $M a-$ durez es muy baja, pero con un grado variable de incertidumbre sobre su verdadero valor. Supongamos además que el objetivo de elaboración es obtener un valor de frutado lo más cercano a 4 como sea posible, y el mayor valor posible de Estado de Batido una vez alcanzado el objetivo de Frutado.

Las Tablas 1 y 2 muestran las referencias sugeridas para Tiempo de Batido y Temperatura de Batido - las variables cuyo valor se debe decidir, el resto de variables de consideran fijas - y los valores de las salidas predichas por el
Cuadro 2: Referencias y valores de salida esperados para el modelado no unitario sin normalización de $S_{f}^{n f}$ para valores dados de $\sigma$.

\begin{tabular}{lllll}
$\sigma$ & 0 & 0.05 & 0.25 & 0.5 \\
\hline Madurez & 4.00 & 4.00 & 4.00 & 4.00 \\
Frutado & 3.95 & 3.94 & 3.95 & 3.93 \\
Estado de Batido & 3.06 & 3.06 & 3.07 & 3.04 \\
Defecto & 1.00 & 1.00 & 1.00 & 1.00 \\
Tiempo de Batido & 3.87 & 3.94 & 4.00 & 3.75 \\
Temperatura de Batido & 2.00 & 2.00 & 2.00 & 2.00
\end{tabular}

modelo para el caso en que $S_{f}^{n f}$ es normalizado y cuando no se normaliza, respectivamente.

Como se observa claramente del Cuadro 1, a medida que $\sigma$ se incrementa, el enfoque normalizado $S_{f}^{n f}$ proporciona valores más conservadores para asegurar que el objetivo de Frutado se alcanza, esto es, reduce Tiempo de Batido y Temperatura de Batido, lo que tiene sentido intuitivamente. Si existe incertidumbre sobre el valor de una entrada, y el objetivo de producción definitivamente prima que Frutado alcance un cierto umbral, la elección natural es ser conservador en la selección de las referencias para asegurar alcanzar el objetivo principal a costa del secundario.

Por otro lado, el enfoque no normalizado (Cuadro 2) inicialmente incremental el valor de Tiempo de Batido, lo que 
es contraituitivo y no la acción que un operador experto haría. Esto es debido a la modificación del peso asignado al arco que relaciona Madurez y Frutado debido a la activación de un mayor número de reglas a un mayor nivel para el nodo. El efecto es equivalente a considerar que la influencia sobre Frutado de tener un cierto valor de Madurez se incrementa. Dado que el valor de Madurez es favorable para alcanzar buenos valores de Frutado, entonces existe mayor posibilidad de perseguir el objetivo secundario, lo que implica incrementar Tiempo de Batido. Este efecto se puede visualizar en la Figura 7, que muestra Frutado como función de Temperatura de Batido y Madurez para diferentes valores de incertidumbre para esta última variable. Los gráficos claramente muestran el incremento de peso asignado a Madurez en el enfoque no normalizado, visible en los valores más alto de Frutado asignados a valores bajos de Madurez para valores altos de Temperatura de Batido (marcado con una X en el gráfico).

\section{Conclusiones}

En este trabajo se han analizado las implicaciones de utilizar emborronamiento no unitario para modelos FCM y su aplicación a un DSS para el PEAOV. Se mostrado que normalizar $S_{f, n s}$ proporciona valores que se corresponden mejor con las decisiones que un operador experto haría y se ha interpretado el por qué de este efecto.

Las líneas de trabajo futuro tratarán sobre si el resultado obtenido es específico para FCM debido a su metodología de modelado particular, o si es también aplicable a FLS basados en reglas estándar. Otra línea de trabajo incluye el análisis del impacto de utilizar diferentes reglas para el modelado de la interacción entre las entradas al sistema y los antecedentes, en particular para conjuntos borrosos no simétricos. Finalmente se está considerando explorar variantes de emborronamiento no unitario que pueden proporcionar una mejor captura de la interacción entre la entrada y el antecedente [9].

\section{Agradecimientos}

Este trabajo ha sido parcialmente subvencionado por los proyectos DPI2011-27284, TEP2009-5363 y AGR-6429. P. Cano Marchal ha sido beneficiario de una beca F.P.U. del Ministerio de Educación del Gobierno de España.

\section{Referencias}

[1] J.H. Aladi, C. Wagner, and J.M. Garibaldi. Type-1 or interval type-2 fuzzy logic systems \#x2014; On the relationship of the amount of uncertainty and FOU size. In 2014 IEEE International Conference on Fuzzy Systems (FUZZ-IEEE), pages 2360-2367, July 2014.
[2] E. F. Camacho and Carlos Bordons. Model predictive control. Springer, 2004.

[3] P. Cano Marchal, J. Gámez Garcia, and J. Gómez Ortega. Decision Support System Based on Fuzzy Cognitive Maps and Run-to-Run Control for Global SetPoint Determination. In 2015 IEEE International Conference on Systems, Man, and Cybernetics (SMC), pages 1745-1751, October 2015.

[4] P. Cano Marchal, J. Gómez Ortega, D. Aguilera Puerto, and J. Gámez García. Situación actual y perspectivas futuras del control del proceso de elaboración del aceite de oliva virgen. Revista Iberoamericana de Automática e Informática Industrial RIAI, 8(3):258-269, July 2011.

[5] Bart Kosko. Fuzzy Cognitive Maps. International Journal of Man-Machine Studies, 24:65-74, 1986.

[6] Jerry M. Mendel. Uncertain Rule-Based Fuzzy Logic Systems: Introduction and New Directions. Prentice Hall, Upper Saddle River, NJ, 2001.

[7] Yuan Miao, Chunyan Miao, XueHong Tao, Zhiqi Shen, and ZhiQiang Liu. Transformation of Cognitive Maps. IEEE Transactions on Fuzzy Systems, 18(1):114-124, February 2010.

[8] G.C. Mouzouris and J.M. Mendel. Nonsingleton fuzzy logic systems: theory and application. IEEE Transactions on Fuzzy Systems, 5(1):56-71, February 1997.

[9] A. Pourabdollah, C. Wagner, and J. Aladi. Changes under the hood - a new type of non-singleton fuzzy logic system. In 2015 IEEE International Conference on Fuzzy Systems (FUZZ-IEEE), pages 1-8, August 2015 .

[10] C.D. Stylios and Peter P. Groumpos. Modeling complex systems using fuzzy cognitive maps. IEEE Transactions on Systems, Man and Cybernetics, Part A: Systems and Humans, 34(1):155-162, January 2004.

[11] C. Wagner and H. Hagras. Toward General Type-2 Fuzzy Logic Systems Based on zSlices. IEEE Transactions on Fuzzy Systems, 18(4):637-660, August 2010. 